\title{
Erratum: Correction to Authors Names and Erratum Institutional Affiliation
}

Kim JY. Relationships between dietary habits and allostatic load index in metabolic syndrome patients. Korean J Fam Med 2013;34: 334-346. http://dx.doi.org/10.4082/kjfm.2013.34.5.334

To the Editor:

In the articles titled "Relationships between Dietary Habits and Allostatic Load Index in Metabolic Syndrome Patients" that appeared in the September 2013 issue of Korean Journal of Family Medicine (Korean J Fam Med 2013;34:334-346), we found that the names and the institutional affiliation of the authors were published incorrectly.

Ju Young Kim*

Department of Family Medicine, Seoul National University Bundang Hospital, Seoul National University College of Medicine, Seongnam, Korea

This is corrected as follows:

Kee Hyuck Lee ${ }^{\dagger}$, Sang Wook Park ${ }^{1, \dagger}$, Sung Min Ye ${ }^{1}$, So-Yeon Kim ${ }^{1}$, Sun-Young Kim ${ }^{1}$, Jong Soo Han, Sarah Kim, Woo Kyung Bae, Ki Heon Lee, Ju Young Kim*

Department of Family Medicine, Seoul National University Bundang Hospital, Seoul National University College of Medicine, Seongnam; ${ }^{1}$ Department of Family Medicine, Seoul National University Hospital, Seoul National University College of Medicine, Seoul, Korea

${ }^{+}$These authors contributed equally to this work.

Korean Journal of Family Medicine

Copyright (C) 2013 The Korean Academy of Family Medicine

(c) This is an open-access article distributed under the terms of the Creative Commons Attribution Non-Commercial License (http:// creativecommons.org/licenses/by-nc/3.0) which permits unrestricted noncommercial use, distribution, and reproduction in any medium, provided the original work is properly cited. 